Vietnam Journal of Mechanics, VAST, Vol.39, No.3 (2017), pp. 215-228

DOI:10.15625/0866-7136/8631

\title{
MODE SHAPE ANALYSIS OF MULTIPLE CRACKED FUNCTIONALLY GRADED BEAM-LIKE STRUCTURES BY USING DYNAMIC STIFFNESS METHOD
}

\author{
Tran Van Lien ${ }^{1, *}$, Ngo Trong Duc ${ }^{2}$, Nguyen Tien Khiem ${ }^{3}$ \\ ${ }^{1}$ National University of Civil Engineering, Hanoi, Vietnam \\ ${ }^{2}$ Design Consultant and Investment of Construction - Ministry of Defense, Vietnam \\ ${ }^{3}$ Institute of Mechanics, Vietnam Academy of Science and Technology, Hanoi, Vietnam \\ *E-mail: lientv@hotmail.com \\ Received August 20, 2016
}

\begin{abstract}
Mode shapes of multiple cracked beam-like structures made of Functionally Graded Material (FGM) are analyzed by using the dynamic stiffness method. Governing equations in vibration theory of multiple cracked FGM beam are derived on the base of Timoshenko beam theory; power law variation of material; coupled spring model of crack and taking into account the actual position of neutral axis. A general solution of vibration in frequency domain is obtained and used for constructing dynamic stiffness matrix of the multiple cracked FGM Timoshenko beam element that provides an efficient method for modal analysis of multiple cracked FGM frame structures. The theoretical development is illustrated by numerical analysis of crack-induced change in mode shapes of multi-span continuous FGM beam.
\end{abstract}

Keywords: FGM, multiple cracked beam, modal analysis, dynamic stiffness method.

\section{INTRODUCTION}

Recently, vibration of cracked FGM structures attracts enormous attention of researchers in the field of structural engineering. Most of the studies rely on the basics of fracture mechanics [1] that allows various models of crack in a structural member to be established [2]. Using the equivalent spring model of cracks, numerous methods such as analytical method, Galerkin and Ritz method, Finite Element Method (FEM) have been developed for analysis of cracked FGM beams.

Yan et al. [3] calculated natural frequencies and mode shapes of cracked FGM Euler-Bernoulli beam using the analytical method. Ke et al. [4] studied effects of open edge cracks to vibration of FGM Timoshenko beam with different boundary conditions. Aydin [5] established frequency equations of free vibration for FGM Euler-Bernoulli beam

(C) 2017 Vietnam Academy of Science and Technology 
with arbitrary number of cracks. Wei et al. [6] used the transfer matrix method to obtain frequency equations and mode shapes of beam with arbitrary number of open edge cracks only in the form of third-order determinant. This is remarkable improvement in order to study free vibration of multiple cracked FGM beam. Sherafatnia et al. [7] analyzed natural frequencies and mode shapes of cracked beam according to Euler-Bernoulli, Rayleigh, shear deformation and Timoshenko theories. The results show that the fundamental mode shapes calculated from the beam theories are identical, but mode shapes of higher modes from the second one are different. Yan et al. [8] obtained dynamic deflections of cracked FGM beam on elastic foundation under a transverse moving load. Besides, Kitipornchai et al. [9] used Ritz's method to analyze nonlinear vibration of FGM Timoshenko beam with an open edge crack. Wattanasakulpong et al. [10] investigated free vibration of FGM beams with general elastically end constraints by differential transformation method. Akbas [11] studied free vibration and wave propagation analysis of a cracked FGM cantilever beam. However, these results were only applied to EulerBernoulli beam. The authors of Refs. [12,13] used FEM and so-called frequency contour method for identification of location and size of cracks in FGM beam. An analytical method is proposed in [14] for identification of an open crack in FGM beam by measurements of three natural frequencies.

As the FEM is formulated by using frequency independent polynomial shape function, the FEM cannot capture all necessary high frequencies and mode shapes of interest. An alternative approach that enables to overcome the drawback of FEM is so-called Dynamic Stiffness Method (DSM). This method was developed for analysis of free vibration of FGM beams in $[15,16]$. The basics of DSM applied for free vibration analysis of FGM beam with single crack were given in $[17,18]$. The present study deals with development of the DSM for modal analysis of multiple cracked beam-like FGM structures.

First, governing equations in vibration theory of FGM Timoshenko beam are derived taking into account the actual position of neutral axis. Then, general solution of free vibration problem is obtained for multiple cracked FGM Timoshenko beam element and used for constructing dynamic stiffness matrix of the beam element. Finally, a new form of frequency equation and an explicit expression for mode shapes of the beam are conducted and employed for mode shape analysis of cracked multi-span continuous FGM beam.

\section{GOVERNING EQUATIONS}

Consider a FGM beam of length $L$, cross sectional area $A=b \times h$. It is assumed that the material properties of FGM beam vary along the thickness by the power law

$$
\left\{\begin{array}{c}
E(z) \\
G(z) \\
\rho(z)
\end{array}\right\}=\left\{\begin{array}{c}
E_{b} \\
G_{b} \\
\rho_{b}
\end{array}\right\}+\left\{\begin{array}{c}
E_{t}-E_{b} \\
G_{t}-G_{b} \\
\rho_{t}-\rho_{b}
\end{array}\right\}\left(\frac{z}{h}+\frac{1}{2}\right)^{n}, \quad-h / 2 \leq z \leq h / 2,
$$

where $E, G$ and $\rho$ stand for Young's, shear modulus and material density, subscripts $t$ and $b$ denote the top and bottom materials, $n$ is power law exponent, $z$ is co-ordinate of point from the mid plane (Fig. 1). Based on the Hamilton's principle, we can get free vibration 


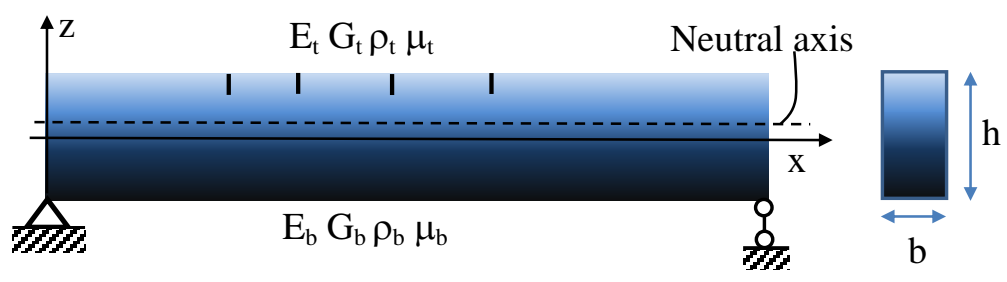

Fig. 1. A multiple cracked FGM beam

equations of FGM Timoshenko beam in frequency domain in the form [17]

$$
[\mathbf{A}]\left\{\mathbf{z}^{\prime \prime}\right\}+[\boldsymbol{\Pi}]\left\{\mathbf{z}^{\prime}\right\}+[\mathbf{D}]\{\mathbf{z}\}=\{\mathbf{0}\},
$$

where $\{\mathbf{z}\}$ is amplitude of axial displacement, slope and deflection

$$
\{\mathbf{z}\}=\{U, \Theta, W\}^{T}=\int_{-\infty}^{\infty}\left\{u_{0}(x, t), \theta(x, t), w_{0}(x, t)\right\}^{T} e^{-i \omega t} d t
$$

$[\mathbf{A}],[\Pi],[\mathbf{D}]$ are matrices

$$
[\mathbf{A}]=\left[\begin{array}{ccc}
A_{11} & -A_{12} & 0 \\
-A_{12} & A_{22} & 0 \\
0 & 0 & A_{33}
\end{array}\right],[\mathbf{\Pi}]=\left[\begin{array}{ccc}
0 & 0 & 0 \\
0 & 0 & A_{33} \\
0 & -A_{33} & 0
\end{array}\right],[\mathbf{D}]=\left[\begin{array}{ccc}
\omega^{2} I_{11} & -\omega^{2} I_{12} & 0 \\
-\omega^{2} I_{12} & \omega^{2} I_{22}-A_{33} & 0 \\
0 & 0 & \omega^{2} I_{11}
\end{array}\right],
$$

and

$$
\begin{aligned}
& \left(A_{11}, A_{12}, A_{22}\right)=\int_{A} E(z)\left(1, z-h_{0},\left(z-h_{0}\right)^{2}\right) d A, A_{33}=\eta \int_{A} G(z) d A, \\
& \left(I_{11}, I_{12}, I_{22}\right)=\int_{A} \rho(z)\left(1, z-h_{0},\left(z-h_{0}\right)^{2}\right) d A, \\
& h_{0}=\frac{n\left(R_{E}-1\right) h}{2(n+2)\left(n+R_{E}\right)}, R_{E}=\frac{E_{t}}{E_{b}}, \eta=5 / 6 .
\end{aligned}
$$
equation

Seeking solution of Eq. (2) in the form $\mathbf{z}_{0}=\mathbf{d} e^{\lambda x}$, we obtain so-called characteristic

$$
\operatorname{det}\left(\lambda^{2}[\mathbf{A}]+\lambda[\mathbf{\Pi}]+[\mathbf{D}]\right)=0 .
$$

This is a cubic algebraic equation respect to $s=\lambda^{2}$ that gives three roots $s_{1}, s_{2}, s_{3}$. Therefore, we get

$$
\lambda_{1,4}= \pm k_{1}, \lambda_{2,5}= \pm k_{2}, \quad \lambda_{3,6}= \pm k_{3}, k_{j}=\sqrt{s_{j}}, j=1,2,3 .
$$

Hence, general solution of Eq. (2) can be obtained in the form [17]

$$
\left\{\mathbf{z}_{0}(x, \omega)\right\}=[\mathbf{G}(x, \omega)]\{\mathbf{C}\},
$$


where $\mathbf{C}=\left(C_{1}, \ldots, C_{6}\right)^{T}$ are constants, $[\mathbf{G}(x, \omega)]$ is matrix

$$
[\mathbf{G}(x, \omega)]=\left[\begin{array}{cccccc}
\alpha_{1} e^{k_{1} x} & \alpha_{2} e^{k_{2} x} & \alpha_{3} e^{k_{3} x} & \alpha_{1} e^{-k_{1} x} & \alpha_{2} e^{-k_{2} x} & \alpha_{3} e^{-k_{3} x} \\
e^{k_{1} x} & e^{k_{2} x} & e^{k_{3} x} & e^{-k_{1} x} & e^{-k_{2} x} & e^{-k_{3} x} \\
\beta_{1} e^{k_{1} x} & \beta_{2} e^{k_{2} x} & \beta_{3} e^{k_{3} x} & -\beta_{1} e^{-k_{1} x} & -\beta_{2} e^{-k_{2} x} & -\beta_{3} e^{-k_{3} x}
\end{array}\right],
$$

and

$$
\alpha_{j}=\frac{\omega^{2} I_{12}}{\omega^{2} I_{11}+\lambda_{j}^{2} A_{11}}, \quad \beta_{j}=\frac{\lambda_{j} A_{33}}{\left(\omega^{2} I_{11}+\lambda_{j}^{2} A_{33}\right)}, j=1,2, \ldots, 6 .
$$

It is assumed that the beam has been cracked at different positions $e_{1}, \ldots, e_{n}$ as shown in Fig. 2(a) and the cracks are open and perpendicular to beam surface. Since axial and transverse vibrations are generally coupled in FGM beam, the coupled spring model (rotational and linear of stiffness $k_{j}^{Y}, k_{j}^{X}$ ) shown in Fig. 2(b) is adopted.

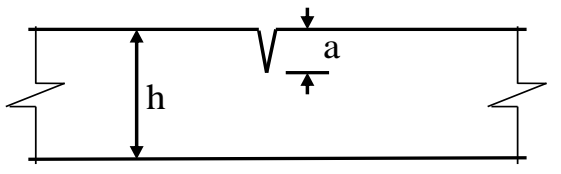

(a)

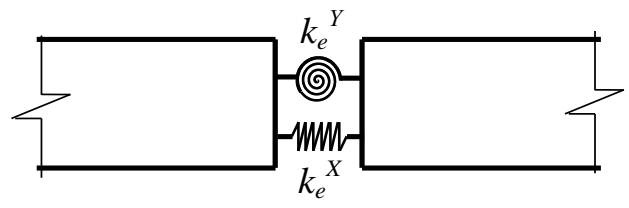

(b)

Fig. 2. A FGM beam with an open edge crack

Therefore, conditions that must be satisfied at a crack are [17]

$$
\begin{aligned}
& U(e+0)-U(e-0)=N(e) / k_{e}^{X}, \Theta(e+0)-\Theta(e-0)=M(e) / k_{e}^{Y}, \\
& W(e+0)=W(e-0), N(e)=N(e+0)=N(e-0), \\
& Q(e+0)=Q(e-0), M(e+0)=M(e-0)=M(e),
\end{aligned}
$$

where $N, Q$ and $M$ are internal axial, shear forces and bending moment respectively

$$
N=A_{11} U_{x}^{\prime}-A_{12} \Theta^{\prime}, \quad M=A_{12} U_{x}^{\prime}-A_{22} \Theta_{x}^{\prime}, \quad Q=A_{33}\left(W_{x}^{\prime}-\Theta\right) \text {. }
$$

Substituting (11) into (10) in case of $A_{12}=0$, we can rewrite the conditions (10) as follows

$$
\begin{aligned}
& U(e+0)=U(e-0)+\gamma_{1} U_{x}^{\prime}(e), \Theta(e+0)=\Theta(e-0)+\gamma_{2} \Theta_{x}^{\prime}(e), \\
& W(e+0)=W(e-0), U_{x}^{\prime}(e+0)=U_{x}^{\prime}(e-0), \\
& \Theta_{x}^{\prime}(e+0)=\Theta_{x}^{\prime}(e-0), W_{x}^{\prime}(e+0)=W_{x}^{\prime}(e-0)+\gamma_{2} \Theta_{x}^{\prime}(e),
\end{aligned}
$$

where

$$
\gamma_{1}=A_{11} / k_{e}^{X}, \quad \gamma_{2}=A_{22} / k_{e}^{Y}
$$

The so-called crack magnitudes $\gamma_{1}, \gamma_{2}$ introduced in (13) are functions of the material properties such as Young's modulus, power law exponent $n$ and cross sectional dimensions. For the FGM beam, the crack magnitudes can be represented in the form [14]

$$
\gamma_{1}=\gamma_{a} \sigma_{1}\left(R_{E}, n\right), \quad \gamma_{2}=\gamma_{b} \sigma_{2}\left(R_{E}, n\right),
$$


where

$\gamma_{a}=E_{0} A / T, \gamma_{b}=E_{0} I / R, I=b h^{3} / 12, E_{0}=\left(E_{t}+E_{b}\right) / 2$,
$\sigma_{1}\left(R_{E}, n\right)=\frac{2\left(R_{E}+n\right)}{\left(R_{E}+1\right)(1+n)}, \sigma_{2}\left(R_{E}, n\right)=\frac{24}{R_{E}+1}\left(\frac{3 R_{E}+n}{3(3+n)}-\frac{2 R_{E}+n}{2+n} \alpha+\frac{R_{E}+n}{1+n} \alpha^{2}\right)$.

In case of homogenous beam $E_{t}=E_{b}=E_{0}\left(R_{E}=1\right)$, the crack magnitudes must be equal to $\gamma_{10}, \gamma_{20}$ that are calculated from crack depth $a_{j}$ for axial [19] and flexural [20] vibration as

$$
\begin{aligned}
& \gamma_{10}=E_{0} A / T_{0}=2 \pi\left(1-v^{2}\right) h f_{1}(z), \quad z=a / h \\
& f_{1}(z)= z^{2}\left(0.6272-0.17248 z+5.92134 z^{2}-10.7054 z^{3}+31.5685 z^{4}-67.47 z^{5}+\right. \\
&\left.+139.123 z^{6}-146.682 z^{7}+92.3552 z^{8}\right), \\
& \gamma_{20}= E_{0} I / R_{0}=6 \pi\left(1-v^{2}\right) h f_{2}(z), \\
& f_{2}(z)= z^{2}\left(0.6272-1.04533 z+4.5948 z^{2}-9.9736 z^{3}+20.2948 z^{4}-33.0351 z^{5}+\right. \\
&\left.+47.1063 z^{6}-40.7556 z^{7}+19.6 z^{8}\right) .
\end{aligned}
$$

Therefore, for modal analysis of cracked FGM beam, crack magnitudes are proposed to be approximately calculated using expression (14) with $\gamma_{1}=\gamma_{10}$ and $\gamma_{2}=$ $\gamma_{20}$ i.e.

$$
\gamma_{1}=F_{1}(z)=2 \pi\left(1-v^{2}\right) h \sigma_{1} f_{1}(z), \gamma_{2}=F_{2}(z)=6 \pi\left(1-v^{2}\right) h \sigma_{2} f_{2}(z)
$$

These functions would be used below for determining the springs stiffness from given crack depth.

\section{GENERAL SOLUTION OF MULTIPLE CRACKED FGM TIMOSHENKO BEAM IN FREQUENCY DOMAIN}

First, solution $\left\{\mathbf{z}_{c}(x)\right\}$ of Eq. (2) satisfying the initial conditions

$$
\left\{\mathbf{z}_{c}(0)\right\}=\left(\gamma_{1} U_{x}^{\prime}(e), \gamma_{2} \Theta_{x}^{\prime}(e), 0\right)^{T}, \quad\left\{\mathbf{z}_{c}^{\prime}(0)\right\}=\left(0,0, \gamma_{2} \Theta_{x}^{\prime}(e)\right)^{T},
$$

is sought by using expression (7) and it can be represented as [17]

$$
\left\{\mathbf{z}_{c}(x)\right\}=\left[\mathbf{G}_{c}(x)\right]\left\{\mathbf{z}_{0}^{\prime}(e)\right\},
$$

where

$$
\left[\mathbf{G}_{c}(x)\right]=[\mathbf{L}(x)][\boldsymbol{\Sigma}],\left\{\mathbf{z}_{0}^{\prime}(e)\right\}=\left(U_{0}^{\prime}(e) \Theta_{0}^{\prime}(e) W_{0}^{\prime}(e)\right)^{T},
$$

and

$$
\begin{gathered}
{[\mathbf{L}(x)]=\left[\begin{array}{ccc}
\alpha_{1} \cosh k_{1} x & \alpha_{2} \cosh k_{21} x & \alpha_{3} \cosh k_{3} x \\
\cosh k_{1} x & \cosh k_{21} x & \cosh k_{3} x \\
\beta_{1} \sinh k_{1} x & \beta_{2} \sinh k_{2} x & \beta_{3} \sinh k_{3} x
\end{array}\right] \cdot\left[\begin{array}{ccc}
\delta_{11} & \delta_{12} & \delta_{13} \\
\delta_{21} & \delta_{22} & \delta_{23} \\
\delta_{31} & \delta_{32} & \delta_{33}
\end{array}\right],[\Sigma]=\left[\begin{array}{ccc}
\gamma_{1} & 0 & 0 \\
0 & \gamma_{2} & 0 \\
0 & \gamma_{2} & 0
\end{array}\right],} \\
\delta_{11}=\left(k_{3} \beta_{3}-k_{2} \beta_{2}\right) / \Delta, \delta_{12}=\left(\alpha_{3} k_{2} \beta_{2}-\alpha_{2} k_{3} \beta_{3}\right) / \Delta, \delta_{13}=\left(\alpha_{2}-\alpha_{3}\right) / \Delta \\
\delta_{21}=\left(k_{1} \beta_{1}-k_{3} \beta_{3}\right) / \Delta, \delta_{22}=\left(\alpha_{1} k_{3} \beta_{3}-\alpha_{3} k_{1} \beta_{1}\right) / \Delta, \delta_{23}=\left(\alpha_{3}-\alpha_{1}\right) / \Delta \\
\delta_{31}=\left(k_{2} \beta_{2}-k_{1} \beta_{1}\right) / \Delta, \delta_{32}=\left(\alpha_{2} k_{1} \beta_{1}-\alpha_{1} k_{2} \beta_{2}\right) / \Delta, \delta_{33}=\left(\alpha_{1}-\alpha_{2}\right) / \Delta \\
\Delta=k_{1} \beta_{1}\left(\alpha_{2}-\alpha_{3}\right)+k_{2} \beta_{2}\left(\alpha_{3}-\alpha_{1}\right)+k_{3} \beta_{3}\left(\alpha_{1}-\alpha_{2}\right) .
\end{gathered}
$$


Using the matrix-function notation

$$
[\overline{\mathbf{G}}(x)]=\left\{\begin{array}{cl}
{\left[\mathbf{G}_{c}(x)\right]} & : x>0 \\
{[\mathbf{0}]} & : x \leq 0
\end{array},\left[\overline{\mathbf{G}}^{\prime}(x)\right]=\left\{\begin{array}{cl}
{\left[\mathbf{G}_{c}^{\prime}(x)\right]} & : x>0 \\
{[\mathbf{0}]} & : x \leq 0
\end{array}\right.\right.
$$

solution of Eq. (2) satisfying conditions (12) can be expressed as

$$
\left\{\mathbf{z}_{c}(x)\right\}=\left\{\mathbf{z}_{0}(x)\right\}+[\overline{\mathbf{G}}(x-e)] \cdot\left\{\mathbf{z}_{0}^{\prime}(e)\right\} .
$$

Similarly, for multiple cracked beam, we get

$$
\begin{aligned}
& \left\{\mathbf{z}_{c}(x)\right\}=\left\{\mathbf{z}_{0}(x)\right\}+\sum_{j=1}^{n}\left[\overline{\mathbf{G}}\left(x-e_{j}\right)\right] \cdot\left\{\boldsymbol{\mu}_{j}\right\}, \\
& \left\{\boldsymbol{\mu}_{j}\right\}=\left\{\mathbf{z}^{\prime}{ }_{0}\left(e_{j}\right)\right\}+\sum_{k=1}^{j-1}\left[\overline{\mathbf{G}}^{\prime}\left(e_{j}-e_{k}\right)\right] \cdot\left\{\boldsymbol{\mu}_{k}\right\}, j=1,2,3, \ldots, n .
\end{aligned}
$$

Substituting (7) into (24), the latter is rewritten as

$$
\left\{\mathbf{z}_{c}(x)\right\}=\left([\mathbf{G}(x, \omega)]+\sum_{j=1}^{n}\left[\overline{\mathbf{G}}\left(x-e_{j}\right)\right] \cdot\left[\tilde{\chi}_{j}\right]\right)\{\mathbf{C}\}=[\mathbf{\Psi}(x, \omega)]\{\mathbf{C}\},
$$

where

$$
\begin{aligned}
& {[\mathbf{\Psi}(x, \omega)]=[\mathbf{G}(x, \omega)]+\sum_{j=1}^{n}\left[\overline{\mathbf{G}}\left(x-e_{j}\right)\right] \cdot\left[\tilde{\chi}_{j}\right],} \\
& {\left[\tilde{\chi}_{j}\right]=\left[\mathbf{G}^{\prime}\left(e_{j}\right)\right]+\sum_{k=1}^{j-1}\left[\overline{\mathbf{G}}^{\prime}\left(e_{j}-e_{k}\right)\right] \cdot\left[\tilde{\chi}_{k}\right], j=1,2,3, \ldots, n .}
\end{aligned}
$$

\section{DYNAMIC STIFFNESS FORMULATION FOR FREE VIBRATION OF MULTIPLE CRACKED FGM TIMOSHENKO BEAM}

Consider the beam element shown in Fig. 3 with nodes labeled $i$ and $j$ at each end of the element

$$
\left\{\hat{\mathbf{U}}_{e}\right\}=\left\{U_{1}, \Theta_{1}, W_{1}, U_{2}, \Theta_{2}, W_{2}\right\}^{T}, \quad\left\{\mathbf{P}_{e}\right\}=\left\{N_{1}, M_{1}, Q_{1}, N_{2}, M_{2}, Q_{2}\right\}^{T},
$$

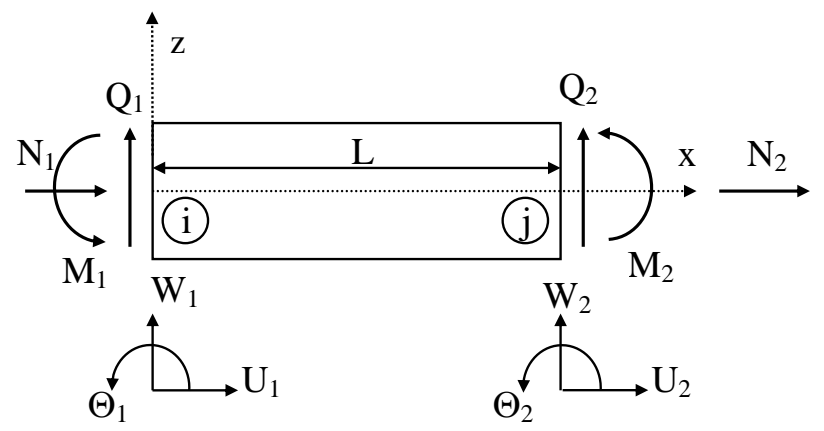

Fig. 3. Beam element and the DOFs 
where

$$
\begin{aligned}
& U_{1}=z_{1}(0, \omega), \Theta_{1}=z_{2}(0, \omega), \quad W_{1}=z_{3}(0, \omega), \\
& U_{2}=z_{1}(L, \omega), \Theta_{2}=z_{2}(L, \omega), \quad W_{2}=z_{3}(L, \omega), \\
& N_{1}=-\left(A_{11} \partial_{x} z_{1}-A_{12} \partial_{x} z_{2}\right)_{x=0}, M_{1}=-\left(A_{22} \partial_{x} z_{2}-A_{12} \partial_{x} z_{1}\right)_{x=0}, Q_{1}=-A_{33}\left(\partial_{x} z_{3}-z_{2}\right)_{x=0}, \\
& N_{2}=\left(A_{11} \partial_{x} z_{1}-A_{12} \partial_{x} z_{2}\right)_{x=L}, M_{2}=\left(A_{22} \partial_{x} z_{2}-A_{12} \partial_{x} z_{1}\right)_{x=L}, Q_{2}=A_{33}\left(\partial_{x} z_{3}-z_{2}\right)_{x=L} .
\end{aligned}
$$

Substituting (28) into (25) yields

$$
\left\{\hat{\mathbf{U}}_{e}\right\}=\left[\begin{array}{c}
{[\mathbf{\Psi}(0, \omega)]} \\
{[\mathbf{\Psi}(L, \omega)]}
\end{array}\right] \cdot\{\mathbf{C}\}, \quad\left\{\mathbf{P}_{e}\right\}=\left[\begin{array}{c}
{\left[-\mathbf{B}_{F}(\mathbf{\Psi})_{x=0}\right]} \\
{\left[\mathbf{B}_{F}(\mathbf{\Psi})_{x=L}\right]}
\end{array}\right] \cdot\{\mathbf{C}\},
$$

where $\left[\mathbf{B}_{F}\right]$ is matrix operator

$$
\left[\mathbf{B}_{F}\right]=\left[\begin{array}{ccc}
A_{11} \partial_{x} & -A_{12} \partial_{x} & 0 \\
A_{12} \partial_{x} & A_{22} \partial_{x} & 0 \\
0 & -A_{33} & A_{33} \partial_{x}
\end{array}\right] .
$$

Eliminating constant vector $\{\mathbf{C}\}$ in (29), we obtain

$$
\left\{\mathbf{P}_{e}\right\}=\left[\begin{array}{c}
{\left[-\mathbf{B}_{F}(\mathbf{\Psi})_{x=0}\right]} \\
{\left[\mathbf{B}_{F}(\mathbf{\Psi})_{x=L}\right]}
\end{array}\right] \cdot\left[\begin{array}{l}
{[\mathbf{\Psi}(0, \omega)]} \\
{[\mathbf{\Psi}(L, \omega)]}
\end{array}\right]^{-1} \cdot\left\{\hat{\mathbf{U}}_{e}\right\}=\left[\hat{\mathbf{K}}_{e}(\omega)\right] \cdot\left\{\hat{\mathbf{U}}_{e}\right\},
$$

where $\left[\hat{\mathbf{K}}_{e}\right]$ is dynamic stiffness matrix of a multiple cracked FGM beam $e$

$$
\left[\hat{\mathbf{K}}_{e}\right]=\left[\begin{array}{c}
{\left[-\mathbf{B}_{F}(\boldsymbol{\Psi})_{x=0}\right]} \\
{\left[\mathbf{B}_{F}(\mathbf{\Psi})_{x=L}\right]}
\end{array}\right] \cdot\left[\begin{array}{l}
{[\mathbf{\Psi}(0, \omega)]} \\
{[\mathbf{\Psi}(L, \omega)]}
\end{array}\right]^{-1} .
$$

Assembling dynamic stiffness matrix (31) for whole structure by the conventional procedure of FEM, free vibration problem for the structure is formulated as

$$
[\hat{\mathbf{K}}(\omega)] \cdot\{\hat{\mathbf{U}}\}=\{\mathbf{0}\} .
$$

Natural frequencies $\{\omega\}=\left\{\begin{array}{llll}\omega_{1} & \omega_{2} & \ldots & \omega_{n}\end{array}\right\}$ are obtained by solving the equation

$$
\operatorname{det}[\hat{\mathbf{K}}(\omega)]=0 \text {. }
$$

Each root $\omega_{j}$ of this equation is related to mode shape

$$
\left\{\phi_{j}(x)\right\}=C_{j}^{0}\left[\mathbf{\Psi}\left(x, \omega_{j}\right)\right]\left[\begin{array}{l}
{[\mathbf{\Psi}(0, \omega)]} \\
{[\mathbf{\Psi}(L, \omega)]}
\end{array}\right]^{-1}\left\{\hat{\mathbf{U}}_{j}\right\},
$$

where $C_{j}^{0}$ is an arbitrary constant, $\left\{\hat{\mathbf{U}}_{j}\right\}$ is the normalized solution of (32) corresponding to $\omega_{j}$.

\section{ANALYSIS OF MODE SHAPES OF MULTIPLE CRACKED FGM TIMOSHENKO BEAM}

\subsection{Numerical validation}

To validate the proposed above theoretical development, natural frequencies and mode shapes of intact and cracked FGM beam are computed and compared to those obtained in earlier studies. 
a) Intact FGM beam: An intact cantilever FGM Timoshenko beam with aluminum $\mathrm{Al}_{2} \mathrm{O}_{3}$ in the top: $E_{t}=390 \mathrm{GPa}, \rho_{t}=3960 \mathrm{~kg} / \mathrm{m}^{3}, \mu_{t}=0.25$ and steel at the bottom: $E_{b}=210 \mathrm{GPa}, \rho_{b}=7800 \mathrm{~kg} / \mathrm{m}^{3}, \mu_{b}=0.31$ is examined for power law exponent $n=1$ and geometry dimensions $L=1.0 \mathrm{~m}, b=0.1 \mathrm{~m}, h=0.1 \mathrm{~m} \mathrm{[16]}$.

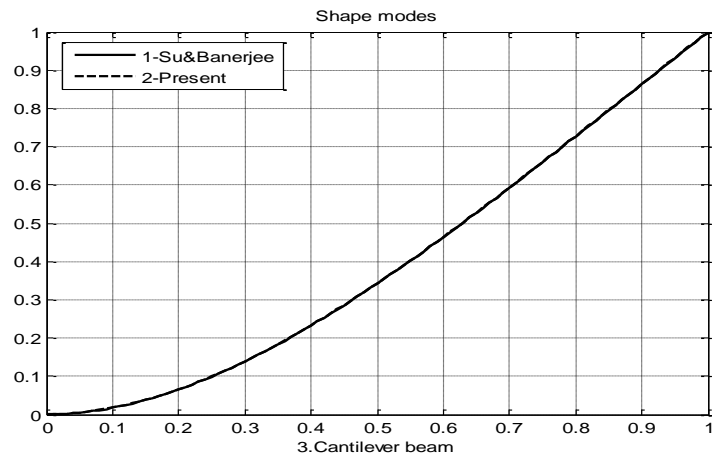

(a)

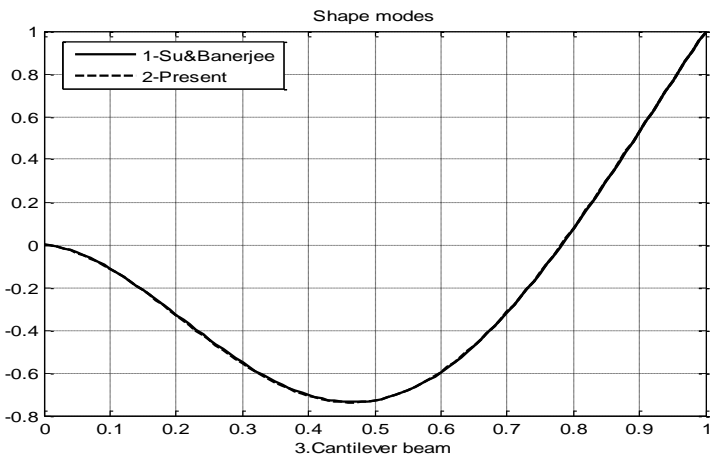

(b)

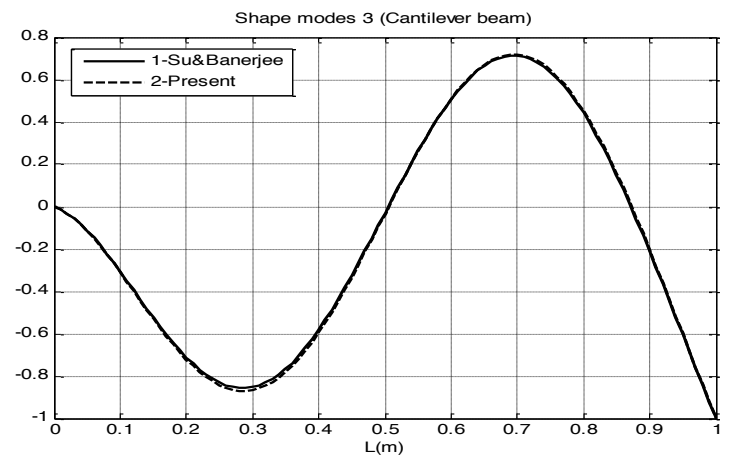

(c)

Fig. 4. Comparing the first three mode shapes of intact cantilever FGM Timoshenko beam with the ones of Su \& Banerjee [16]

Three lowest mode shapes of intact cantilever FGM beam calculated by the above theory are compared with those given in [16] and shown in Fig. 4. There is demonstrated an excellent agreement between the results obtained herein and that that given by Su \& Banerjee.

b) Cracked FGM beam: A FGM beam with aluminum at the top surface: $E_{t}=70$ $\mathrm{GPa}, E_{b} / E_{t}=0.2, \rho_{t}=\rho_{b}=2780 \mathrm{~kg} / \mathrm{m}^{3}, \mu_{t}=0.33$ is examined for beam with power law exponent $n=1$, geometry dimensions $L=2.0 \mathrm{~m}, b=0.1 \mathrm{~m}, h=0.1 \mathrm{~m}$ and a single crack at location $1.0 \mathrm{~m}$ and of the depth $a / h=20 \%$ [3].

Fig. 5 shows the fundamental mode shapes of cracked FGM beam with simple supports, clamped ends and cantilever calculated by the above theory and Yang \& Chen [3]. It is observed also a good agreement of the results and this verifies again validity and usefulness of the proposed above theory. Now we apply it for investigating mode shapes of a FGM beam with rigid supports. 


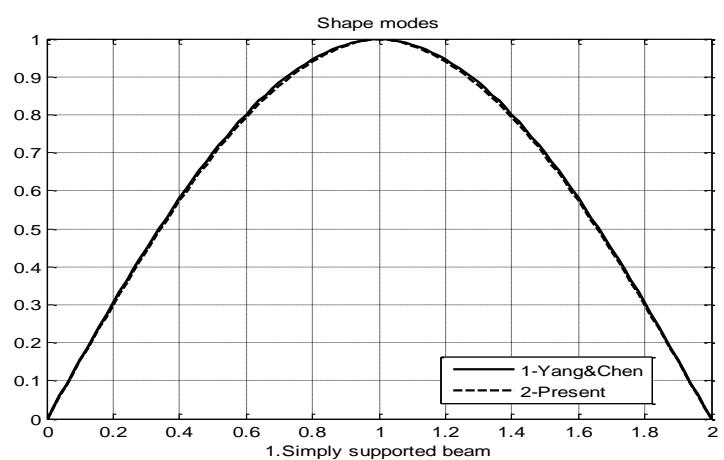

(a) Simple support

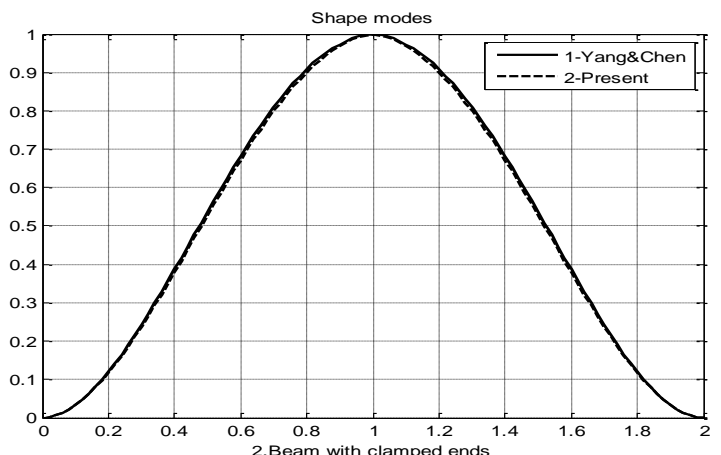

(b) Clamped ends

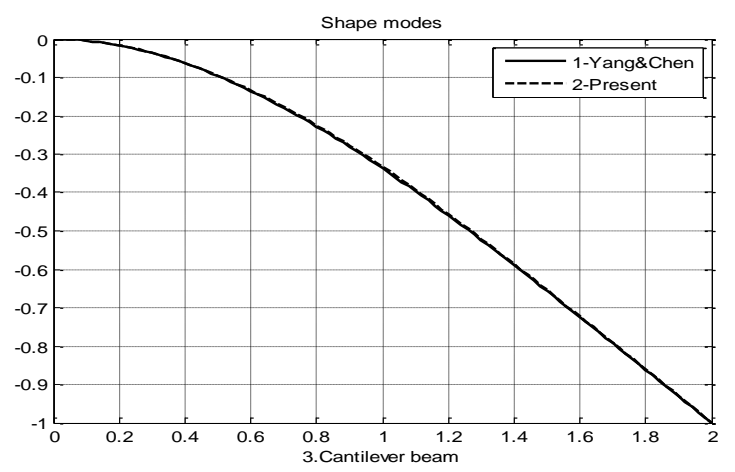

(c) Cantilever

Fig. 5. Comparing the fundamental vibration mode shapes of cracked FGM beam with the ones of Yang \& Chen [3] with different boundary conditions

\subsection{Numerical illustration}

Consider a multiple-span continuous beam with the following parameters: cross section area $b \times h=0.1 \mathrm{~m} \times 0.1 \mathrm{~m}, E_{t}=70 \mathrm{GPa}, \rho_{t}=2780 \mathrm{~kg} / \mathrm{m}^{3}, \mu_{t}=0.33, E_{b}=350$ GPa, $\rho_{b}=7800 \mathrm{~kg} / \mathrm{m}^{3}, \mu_{b}=0.33$ and power law exponent $n=0.5$ (Fig. 6).

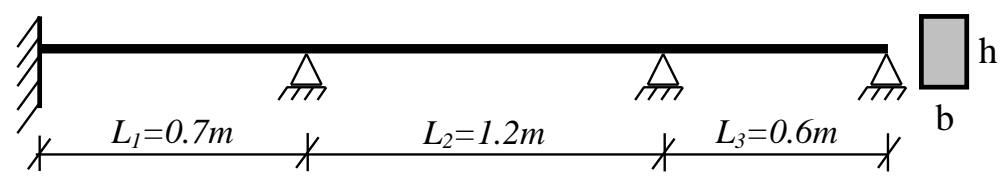

Fig. 6. Multiple-span continuous beam

Figs. 7(a)-7(c) show three lowest mode shapes of multiple-span continuous beam with various numbers of cracks ( 1 to 4 cracks, an equidistance $0.1 \mathrm{~m}$ ) in the second span. The depths of all cracks are 30\%. Fig. 7(d)-7(f) show the deviation of first three mode cracked shapes from the intact ones. It can be seen from the figures that increasing number of cracks leads to change in mode shapes of whole the beam but significant deviation 
is observed at only cracked span, where mode shapes are suddenly changed at the crack positions. On other uncracked spans, the mode shapes are still smooth.

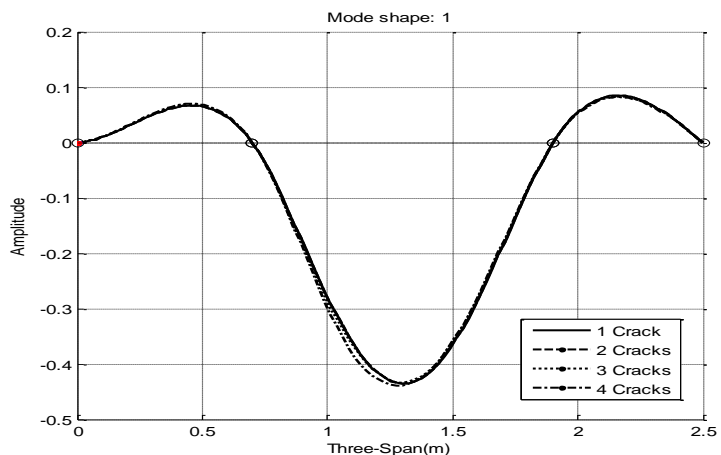

(a)

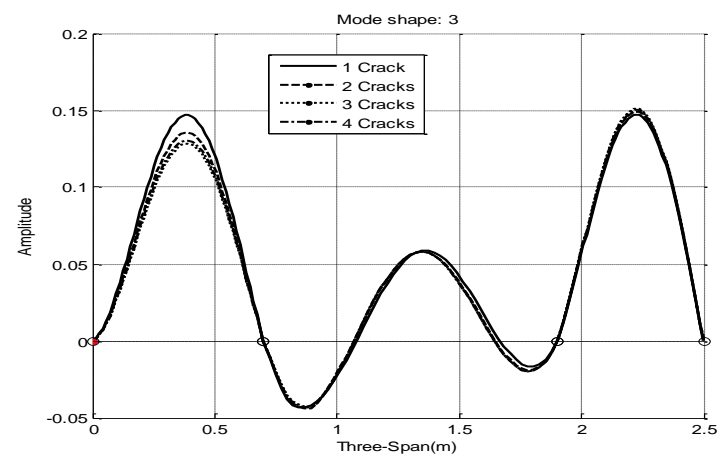

(c)

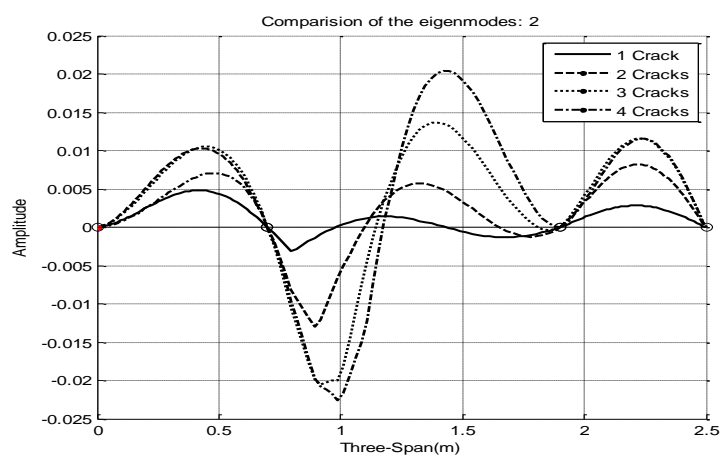

(e)

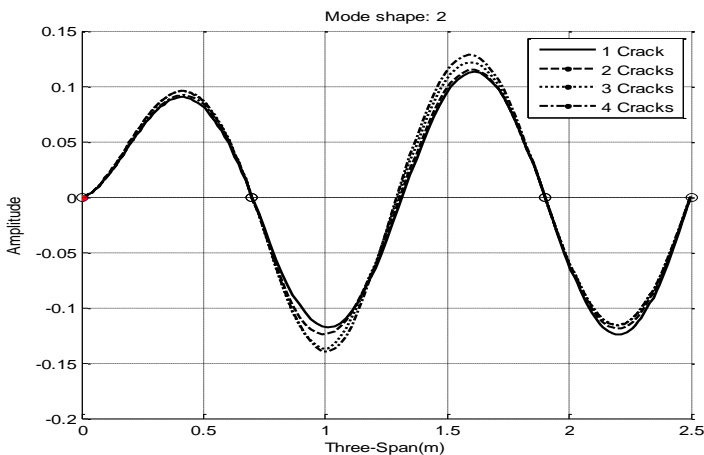

(b)

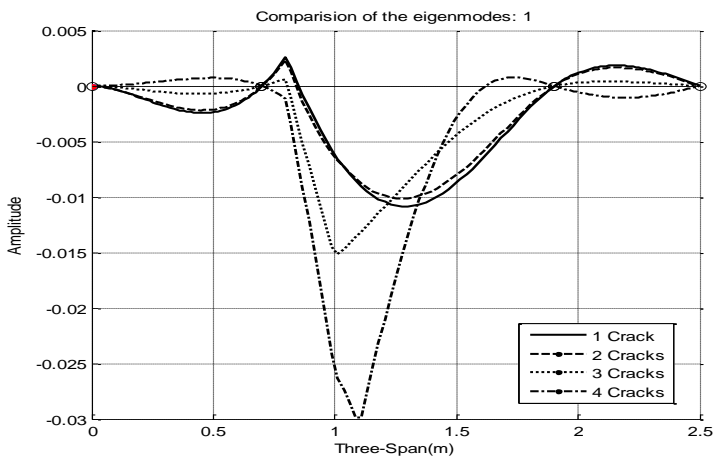

(d)

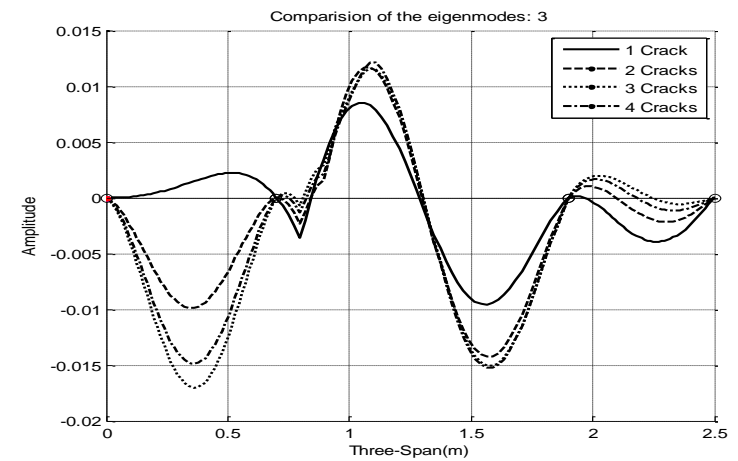

(f)

Fig. 7. Deviation of first three mode shapes of the multi-span continuous FGM beam with various numbers of cracks (from 1 to 4 cracks) in the second span

Fig. 8 show the change in mode shape of first (Figs. 8(a)-8(c)), second (Figs. 8(d)$8(\mathrm{f})$ ) and third (Figs. 8(g)-8(i)) modes of the beam that has 1 crack in the intermediate span. The crack are located at the position $0.2 \mathrm{~m}, 0.3 \mathrm{~m}, 0.4 \mathrm{~m}$ respectively and depth varying from $10 \%$ to $30 \%$. 


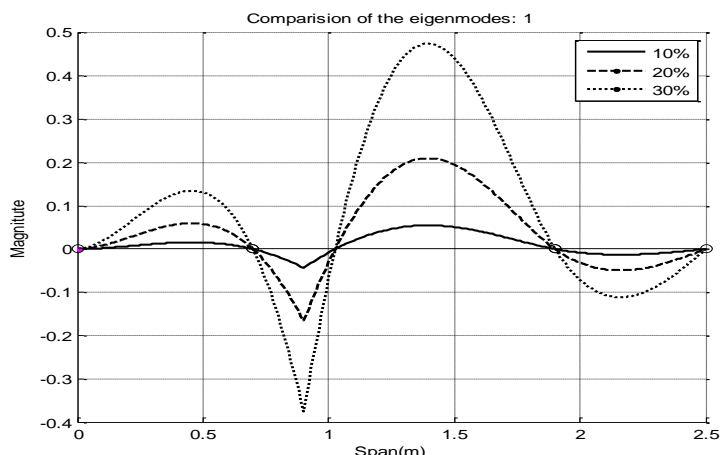

(a)

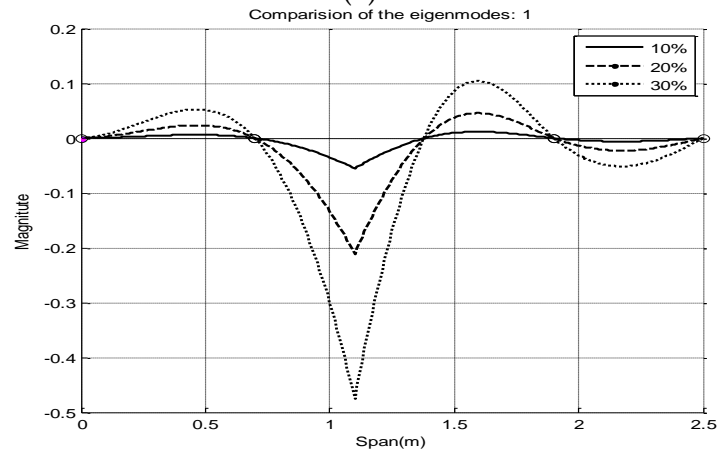

(c)

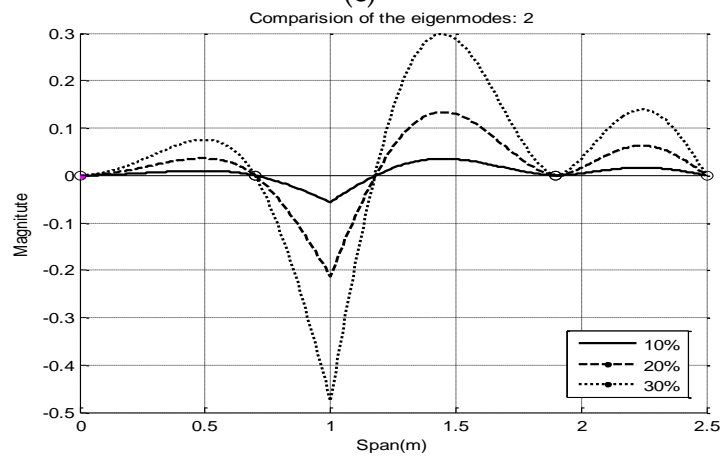

(e)

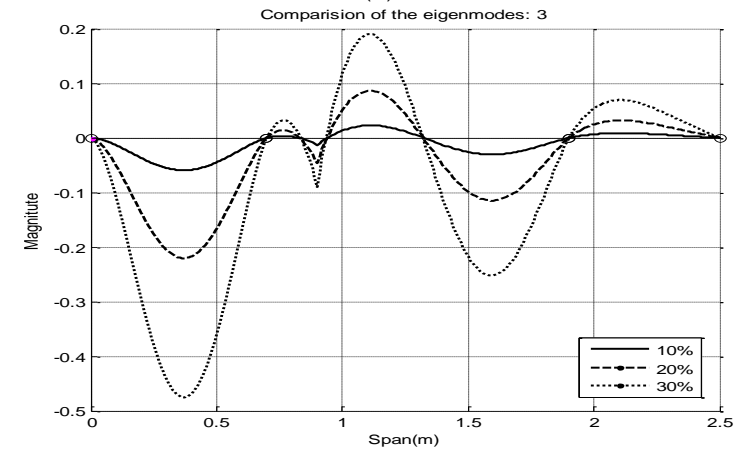

(g)

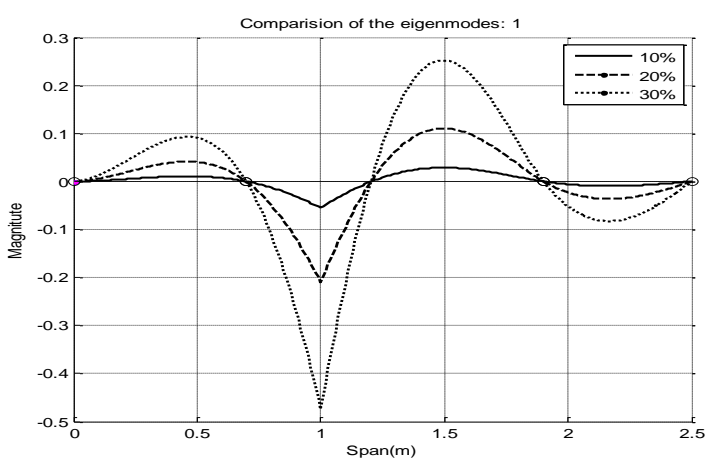

(b)

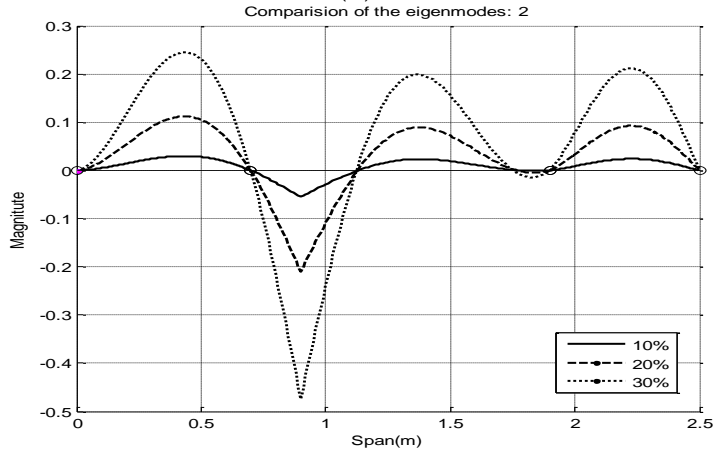

(d)

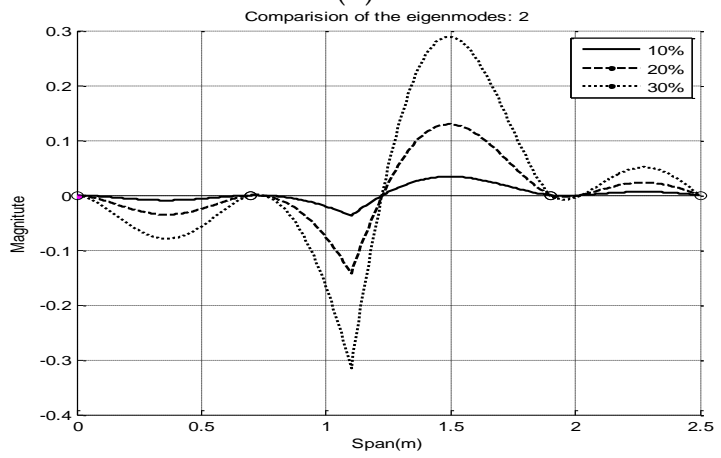

(f)

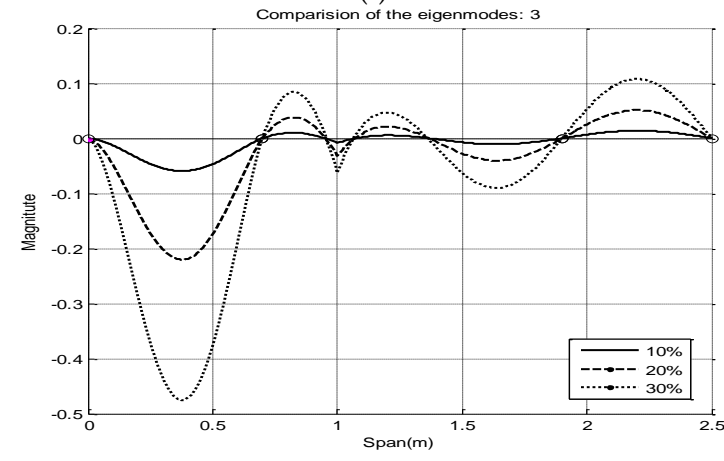

(h) 


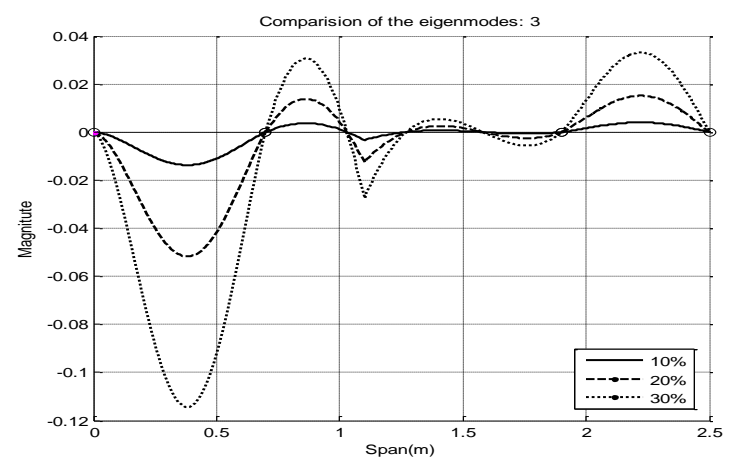

(i)

Fig. 8. Deviation of first three mode shapes of the multiple-span continuous FGM beam with different crack locations in the second span from left node end

Observing graphs in the figures allows one to make some remarks as follows:

- At crack positions, the deviations of the mode shapes reach a peak at the same point of crack position, but it is not maximum value. It is useful indication for localizing crack in the structures by using wavelet transformation of mode shapes.

- The deviation of the mode shapes increases with the increasing crack depth. that enables to estimate also crack depth by wavelet coefficient of mode shape at the crack location.

- At cracked span, the mode shapes change suddenly, while on the other intact spans the mode shapes change smoothly.

- There are some positions in which the cracks do not influence upon the mode shapes, for example: the crack at position $0.2 \mathrm{~m}$ from left node of the second span cause no change in first mode shape at position of $1.05 \mathrm{~m}$ (Fig. 8(a)), second mode shape at position $1.12 \mathrm{~m}$ (Fig. 8(d)) and third mode shape at position $1.35 \mathrm{~m}$ (Fig. 8(g)). Thus, these positions are called the invariable points of the mode shape ad they are differenced from the position where the mode shapes have vanished (zero amplitude).

All the mentioned remarks provide useful tool for crack detection in FGM beamlike structures by measurement of mode shapes.

\section{CONCLUSIONS}

In this article, mode shapes of FGM Timoshenko beam-like structures with multiple cracks are examined using the power law distribution of FGM material, coupled spring model of crack and actual position of neutral axis. The frequency equation and mode shape obtained provides a simple approach to study not only free vibration of the structures but also inverse problem like identification of material and crack parameters in structures.

Numerical study on crack-induced change in first three mode shapes of multiple cracked FGM multi-span continuous beam shows that mode shapes of the beam are dependent on material properties; geometric parameter and also are sensitive to crack. 
Namely, there are suddenly changes in mode shapes at crack positions (sharp leaks) and increasing crack depth leads to larger change in the mode shapes. At cracked span, the mode shapes change suddenly, while the mode shapes change smoothly on the other intact spans. The obtained results are basic for next research to determine number, locations and depth of cracks in beam-like FGM structures.

\section{REFERENCES}

[1] B. H. Wu. The surface crack problem for a plate with functionally graded properties. Journal of Applied Mechanics, 64, (1997), pp. 449-456. doi:10.1115/1.2788914.

[2] A. S. J. Swamidas, X. Yang, and R. Seshadri. Identification of cracking in beam structures using Timoshenko and Euler formulations. Journal of Engineering Mechanics, 130, (11), (2004), pp. 1297-1308. doi:10.1061/(asce)0733-9399(2004)130:11(1297).

[3] J. Yang and Y. Chen. Free vibration and buckling analyses of functionally graded beams with edge cracks. Composite Structures, 83, (1), (2008), pp. 48-60. doi:10.1016/j.compstruct.2007.03.006.

[4] L. L. Ke, J. Yang, S. Kitipornchai, and Y. Xiang. Flexural vibration and elastic buckling of a cracked Timoshenko beam made of functionally graded materials. Mechanics of Advanced Materials and Structures, 16, (6), (2009), pp. 488-502. doi:10.1080/15376490902781175.

[5] K. Aydin. Free vibration of functionally graded beams with arbitrary number of surface cracks. European Journal of Mechanics-A/Solids, 42, (2013), pp. 112-124. doi:10.1016/j.euromechsol.2013.05.002.

[6] D. Wei, Y. Liu, and Z. Xiang. An analytical method for free vibration analysis of functionally graded beams with edge cracks. Journal of Sound and Vibration, 331, (7), (2012), pp. 1686-1700. doi:10.1016/j.jsv.2011.11.020.

[7] K. Sherafatnia, G. Farrahi, and S. A. Faghidian. Analytic approach to free vibration and buckling analysis of functionally graded beams with edge cracks using four engineering beam theories. International Journal of Engineering-Transactions C: Aspects, 27, (6), (2013), pp. 979990. doi:10.5829/idosi.ije.2014.27.06c.17.

[8] T. Yan, S. Kitipornchai, J. Yang, and X. Q. He. Dynamic behaviour of edge-cracked shear deformable functionally graded beams on an elastic foundation under a moving load. Composite Structures, 93, (11), (2011), pp. 2992-3001. doi:10.1016/j.compstruct.2011.05.003.

[9] S. Kitipornchai, L. L. Ke, J. Yang, and Y. Xiang. Nonlinear vibration of edge cracked functionally graded Timoshenko beams. Journal of Sound and Vibration, 324, (3), (2009), pp. 962-982. doi:10.1016/j.jsv.2009.02.023.

[10] N. Wattanasakulpong and V. Ungbhakorn. Free vibration analysis of functionally graded beams with general elastically end constraints by DTM. World Journal of Mechanics, 2, (06), (2012), pp. 297-310. doi:10.4236/wjm.2012.26036.

[11] S. D. Akbaş. Free vibration characteristics of edge cracked functionally graded beams by using finite element method. International Journal of Engineering Trends and Technology, 4, (10), (2013), pp. 4590-4597.

[12] Z. Yu and F. Chu. Identification of crack in functionally graded material beams using the p-version of finite element method. Journal of Sound and Vibration, 325, (1), (2009), pp. 69-84. doi:10.1016/j.jsv.2009.03.010.

[13] A. Banerjee, B. Panigrahi, and G. Pohit. Crack modelling and detection in Timoshenko FGM beam under transverse vibration using frequency contour and response surface model with GA. Nondestructive Testing and Evaluation, 31, (2), (2016), pp. 142-164. doi:10.1080/10589759.2015.1071812. 
[14] N. T. Khiem and N. N. Huyen. A method for crack identification in functionally graded Timoshenko beam. Nondestructive Testing and Evaluation, 32, (3), (2017), pp. 319-341. doi:10.1080/10589759.2016.1226304.

[15] H. Su, J. R. Banerjee, and C. W. Cheung. Dynamic stiffness formulation and free vibration analysis of functionally graded beams. Composite Structures, 106, (2013), pp. 854-862. doi:10.1016/j.compstruct.2013.06.029.

[16] H. Su and J. R. Banerjee. Development of dynamic stiffness method for free vibration of functionally graded Timoshenko beams. Computers $\mathcal{E}$ Structures, 147, (2015), pp. 107-116. doi:10.1016/j.compstruc.2014.10.001.

[17] N. T. Khiem, N. D. Kien, and N. N. Huyen. Vibration theory of FGM beam in the frequency domain. In Proceedings of National Conference on Engineering Mechanics celebrating 35th Anniversary of the Institute of Mechanics, VAST, Vol. 1, (2014), pp. 93-98. (in Vietnamese).

[18] T. V. Lien, N. T. Khiem, and N. T. Duc. Free vibration analysis of functionally graded Timoshenko beam using dynamic stiffness method. Journal of Science and Technology in Civil Engineering, (31), (2016), pp. 19-28.

[19] T. G. Chondros, A. D. Dimarogonas, and J. Yao. Longitudinal vibration of a continuous cracked bar. Engineering Fracture Mechanics, 61, (5), (1998), pp. 593-606. doi:10.1016/s00137944(98)00071-x.

[20] T. G. Chondros, A. D. Dimarogonas, and J. Yao. A continuous cracked beam vibration theory. Journal of Sound and Vibration, 215, (1), (1998), pp. 17-34. doi:10.1006/jsvi.1998.1640. 